Roberto Cilia, MD

Janeth Laguna, MD

Erica Cassani, MD

Emanuele Cereda, MD,

$\mathrm{PhD}$

Nicolò G. Pozzi, MD

Ioannis U. Isaias, MD,

$\mathrm{PhD}$

Manuela Contin,

PharmD

Michela Barichella, MD

Gianni Pezzoli, MD

Correspondence to

Dr. Cilia:

roberto.cilia@gmail.com

\section{Supplemental data} at Neurology.org

\title{
Mucuna pruriens in Parkinson disease
}

\author{
A double-blind, randomized, controlled, crossover study \\ OPEN \\ 回 $\operatorname{ar}^{\mathrm{P}}$
}

\section{ABSTRACT}

Objective: To investigate whether Mucuna pruriens (MP), a levodopa-containing leguminous plant growing in all tropical areas worldwide, may be used as alternative source of levodopa for indigent individuals with Parkinson disease (PD) who cannot afford long-term therapy with marketed levodopa preparations.

Methods: We investigated efficacy and safety of single-dose intake of MP powder from roasted seeds obtained without any pharmacologic processing. Eighteen patients with advanced PD received the following treatments, whose sequence was randomized: (1) dispersible levodopa at $3.5 \mathrm{mg} / \mathrm{kg}$ combined with the dopa-decarboxylase inhibitor benserazide (LD+DDCl; the reference treatment); (2) high-dose MP (MP-Hd; $17.5 \mathrm{mg} / \mathrm{kg})$; (3) low-dose MP (MP-Ld; $12.5 \mathrm{mg} / \mathrm{kg}$ ); (4) pharmaceutical preparation of LD without DDCl (LD-DDCl; $17.5 \mathrm{mg} / \mathrm{kg}$ ); (5) MP plus benserazide (MP+DDCl; $3.5 \mathrm{mg} / \mathrm{kg})$; (6) placebo. Efficacy outcomes were the change in motor response at 90 and 180 minutes and the duration of on state. Safety measures included any adverse event (AE), changes in blood pressure and heart rate, and the severity of dyskinesias.

Results: When compared to LD+DDCl, MP-Ld showed similar motor response with fewer dyskinesias and AEs, while MP-Hd induced greater motor improvement at 90 and 180 minutes, longer $\mathrm{ON}$ duration, and fewer dyskinesias. MP-Hd induced less AEs than LD+DDCl and LD-DDCl. No differences in cardiovascular response were recorded.

Conclusion: Single-dose MP intake met all noninferiority efficacy and safety outcome measures in comparison to dispersible levodopa/benserazide. Clinical effects of high-dose MP were similar to levodopa alone at the same dose, with a more favorable tolerability profile.

\section{ClinicalTrials.gov identifier: NCT02680977. Neurology ${ }^{\circledR}$ 2017;89:432-438}

\section{GLOSSARY}

$\mathbf{A E}=$ adverse event; $\mathbf{A I M S}=$ Abnormal Involuntary Movements Scale; $\mathbf{D D C I}=$ dopa-decarboxylase inhibitor; $\mathbf{D S M}$-IV-TR = Diagnostic and Statistical Manual of Mental Disorders, 4th edition, text revision; MMSE = Mini-Mental State Examination; MP = Mucuna pruriens variant utilis; MP-Hd = high-dose Mucuna pruriens powder; MP-Ld = low-dose Mucuna pruriens powder; PD = Parkinson disease; UPDRS = Unified Parkinson's Disease Rating Scale.

Since its introduction in the late 1960s, levodopa has remained the most effective and gold standard therapy for Parkinson disease (PD). ${ }^{1}$ However, the access to levodopa in low-income areas is still greatly limited, so that the majority of patients remain either undertreated or untreated, with reduction in quality of life and survival. ${ }^{2-5}$ In rural Africa, it is estimated that only $15 \%$ of patients with PD are treated with levodopa. ${ }^{3}$ Mucuna pruriens variant utilis (MP) is a leguminous plant growing spontaneously in tropical/subtropical areas worldwide, whose seeds contain high concentrations of levodopa. ${ }^{6} \mathrm{MP}$-based therapy may become a potential alternative to marketed

\footnotetext{
From the Parkinson Institute (R.C., E. Cassani, M.B., G.P.), ASST Gaetano Pini-CTO, Milan, Italy; Neurology Clinic (J.L.), Clinica Niño Jesus, Santa Cruz, Bolivia; Nutrition and Dietetics Service (E. Cereda), Fondazione IRCCS Policlinico San Matteo, Pavia; Department of Pathophysiology and Transplantation (N.G.P., I.U.I.), LAMB Pierfranco \& Luisa Mariani, University of Milan, Italy; Department of Neurology (N.G.P., I.U.I.), University Hospital Würzburg and Julius-Maximilians-University, Würzburg, Germany; IRCCS-Institute of Neurological Sciences of Bologna (M.C.); and Department of Biomedical and Neuromotor Sciences (M.C.), University of Bologna, Italy.

Go to Neurology.org for full disclosures. Funding information and disclosures deemed relevant by the authors, if any, are provided at the end of the article. The Article Processing Charge was funded by the Fondazione Grigioni per il Morbo di Parkinson.

This is an open access article distributed under the terms of the Creative Commons Attribution-NonCommercial-NoDerivatives License 4.0 (CC BY-NC-ND), which permits downloading and sharing the work provided it is properly cited. The work cannot be changed in any way or used commercially without permission from the journal.
} 
levodopa-based medications in these regions, because it is easy to find at local markets and requires a low-cost preparation method without pharmacologic processing. ${ }^{6}$

Preclinical and clinical studies suggest that MP powder extract may be used to improve parkinsonism with a favorable safety profile. ${ }^{7-11}$ However, it is unclear whether these encouraging data are due to specific properties of MP itself or the pharmacokinetics of levodopa without any dopa-decarboxylase inhibitor (DDCI) as compared to levodopa plus DDCI. It had been suggested that MP may have an intrinsic DDCI-like activity, ${ }^{7,8}$ or even act through a mechanism that is independent of levodopa.' This study aims to investigate whether (1) the efficacy of MP powder is noninferior to levodopa/benserazide, (2) the intake of MP powder at suprathreshold dose is safe, and (3) the effects of MP are similar to those of a pharmaceutical preparation of levodopa alone.

METHODS Standard protocol approvals, registrations, and patient consents. This study was conducted in accordance with good clinical practice and the Declaration of Helsinki. The study protocol was approved by the institutional ethics committee (protocol ID: SBN.SC.013/2015) and written informed consent was obtained from all individuals entering the study. An additional consent was requested for video recording. The study is registered at ClinicalTrials.gov, identifier NCT02680977.

Study design. This is a noninferiority, double-blind, randomized, controlled, crossover, phase $2 \mathrm{~b}$ study addressing the efficacy and safety of acute intake of MP at 2 doses compared to levodopa plus DDCI.

Participants and eligibility criteria. Patients with idiopathic PD according to UK Brain Bank criteria ${ }^{12}$ were recruited in Santa Cruz (Bolivia) by a local neurologist (J.L.) trained in the administration of the instruments used. Eligibility included sustained response to levodopa and presence of motor fluctuations for at least $1 \mathrm{~h} / \mathrm{d}$ during waking hours associated with dyskinesias (peak dose or diphasic). Patients were on stable antiparkinsonian therapy for at least 30 days before recruitment. Exclusion criteria were (1) signs of cognitive impairment (according to DSM-IV-TR criteria or Mini-Mental State Examination [MMSE] <26) limiting the ability to provide written informed consent; (2) clinically significant psychiatric illness; (3) Hoehn \& Yahr stage 5/5; (4) severe unstable medical conditions; and (5) pregnancy.

Interventions. The investigational treatment was MP powder prepared directly from roasted seeds, with neither any pharmaceutical processing nor additional compounds (e.g., additives), as described previously. ${ }^{6}$ This low-cost method had been suggested by the Bolivian neurologist coauthoring this article (J.L.), who has a longstanding experience in the use of MP powder in indigent individuals with PD. Briefly, we roasted MP seeds in a pan for 15 minutes; we then peeled off the teguments and ground the seeds in a small grinder; we finally passed the ground seeds through a sieve to obtain the powder, which we added to water. We measured the content in levodopa of the Bolivian black ecotype (used in the present study) and found it to be $5.7 \%$, with neither harmful antinutrients nor compounds with DDCI-like activity (such as genistein or its precursor genistin, whose presence in MP had been postulated $\left.{ }^{11}\right){ }^{6}$

Following the screening visit, patients were scheduled to attend the clinic 6 times to receive each of the following treatments: (1) marketed levodopa/benserazide (LD+DDCI, $100 \mathrm{mg} / 25 \mathrm{mg}$ dispersible tablets; considered as the reference treatment) at $3.5 \mathrm{mg} / \mathrm{kg}$; (2) high-dose MP powder (MP-Hd; levodopa at $17.5 \mathrm{mg} / \mathrm{kg}$ ); (3) low-dose MP powder (MP-Ld; levodopa at $12.5 \mathrm{mg} / \mathrm{kg}$ ); (4) pharmaceutical preparation of levodopa alone without DDCI (LD-DDCI; $17.5 \mathrm{mg} / \mathrm{kg}$ ) manufactured at a public pharmacy experienced in galenic preparations located in Milan, Italy; (5) MP powder plus benserazide (MP+DDCI; levodopa at $3.5 \mathrm{mg} / \mathrm{kg}$ ); (6) placebo (see below). Levodopa:benserazide ratio was 4:1.

As we aimed to obtain safety data on acute MP intake, we set the reference levodopa dose at the maximum suprathreshold dose we considered tolerable (i.e., LD+DDCI at $3.5 \mathrm{mg} / \mathrm{kg}$ ). The selection of the levodopa dose ratio of 3.5:1 and 5:1 (MP-Ld and MP-Hd, respectively) was based on previous clinical studies comparing levodopa alone vs combined with DDCIs that demonstrated a $60 \%$ to $80 \%$ reduction in levodopa dose with DDCIs. ${ }^{13-19}$ The choice of the 3.5:1 ratio was additionally supported by our previous study exploring the pharmacokinetics of MP. ${ }^{6}$ We investigated the relationship between MP and the presence vs absence of a DDCI by including both levodopa without DDCI and MP plus DDCI.

Randomization and masking. All participants received the 6 treatments in the morning for 6 consecutive days. Allocation to the intervention groups was performed according to a computer-generated randomization list (table e-1 at Neurology.org) and concealment was attained by using sealed envelopes. All participants and the outcome assessor (R.C.) were blind to treatment allocation. All treatments were dissolved in $100 \mathrm{~mL}$ of water. To obtain similar flavor, smell, and color to MP powder, we prepared placebo and active non-MP treatments by adding $5 \mathrm{~g}$ of ground nuts and $1 \mathrm{~g}$ of soluble coffee (figure e-1). This choice was based on patients' report on MP flavor and corroborated by the investigators who personally tasted MP powder (R.C., J.L., G.P.). Before the trial, the concoctions were tried in some patients who were on stable MP therapy to see whether they could distinguish between the various treatments, and they could not tell what was MP and what was not.

Assessments. Clinical workup included (1) rating of motor symptoms by means of the Unified Parkinson's Disease Rating Scale (UPDRS) part $\mathrm{III}^{20}$ and (2) the Abnormal Involuntary Movements Scale (AIMS). ${ }^{21}$ UPDRS parts I, II, IV, and the Hoehn \& Yahr stage were recorded at the baseline visit. Dopamine agonists, $\mathrm{MAO}-\mathrm{B} / \mathrm{COMT}$ inhibitors, anticholinergics, and amantadine were stopped 7 days before the first day of the study (aiming to minimize differences vs low-income countries, where antiparkinsonian medications besides levodopa are rarely available). ${ }^{2-4}$ All patients were assessed in the morning, in the medication-off state after overnight withdrawal of levodopa ( $\geq 12$ hours) and fasting. Patients were observed every 5 minutes after treatment intake to establish when they switched to the clinical on state; thereafter, they were requested to stay at the clinic until they switched back to the off state (UPDRS-III score $\geq 80 \%$ of the baseline off state). Then, patients were allowed to take their home therapy as scheduled with levodopa/carbidopa until 8 PM. 
Motor response (UPDRS-III) and dyskinesias (AIMS) were assessed 90 and 180 minutes after treatment intake by a neurologist experienced in movement disorders (R.C.). Patients were videotaped in the off state, 90 and 180 minutes after treatment intake.

Outcome measures. Efficacy. The primary efficacy outcome measure was the noninferiority of MP as compared to levodopa/benserazide in terms of the percentage of change in UPDRS-III from the baseline off state 90 and 180 minutes after treatment intake. Secondary efficacy outcome measures included the latency to on (defined as the period between treatment intake and the on state) and the duration of on state (defined as the period from the on state to the subsequent off state).

Safety. Safety was assessed over 180 minutes after study medication intake. Assessments included (1) frequency, type, and duration of any adverse event (AE) recorded, (2) changes in blood pressure and heart rate (cardiovascular endpoints), and (3) the severity of dyskinesias.

Statistical analysis. Considering the randomized crossover study design and the noninferiority of MP-Hd compared to LD + DDCI as the primary objective of the trial, we calculated that at least 18 patients should be included to reject the null hypothesis ( $\alpha$ error, $5 \%$; $\beta$ error, 20\%) of a difference higher than $15 \%$ in the percentage of change (SD, 18\%) in UPDRS-III from the baseline off to 90 minutes postdose on state.

Data were analyzed blinded to treatment using the software program SPSS (Windows release 17.0; SPSS Inc., Chicago, IL). A 2 -sided $p$ value of $<0.05$ was set as significant. Descriptive statistics were provided for continuous (mean and SD or median and interquartile range [25th-75th percentile]) and categorica (count and percentage) variables. Paired 2-group comparisons were performed using the McNemar test (categorical variables) and Student $t$ test or Wilcoxon signed-rank test (continuous variables), as appropriate. Primary analysis was focused on the comparisons between single interventions and the reference treatment (levodopa/benserazide). Other paired between-group comparisons were also considered.

RESULTS Eighteen patients (13 male, 5 female) with advanced PD were included in the study, all of whom had motor fluctuations and dyskinesias (table 1). Mean disease duration was 10 years and the mean duration of on time after the single dose of levodopa/carbidopa at home was approximately 160 minutes. Eight patients had been on stable MP therapy for a median period of 3.5 years.

Efficacy. MP-Hd and MP-Ld were noninferior to LD + DDCI in all primary and secondary efficacy outcome measures. Results are shown in figure 1 and presented in detail in table e-2. A paradigmatic case is shown in the video.

Intake of MP-Ld and MP+DDCI provided similar motor responses to $\mathrm{LD}+\mathrm{DDCI}$, while MP-Hd induced a greater improvement in motor symptoms at 90 and 180 minutes $(p=0.037$ and $p=0.002$, respectively). In comparison to motor performance at 90 minutes, UPDRS-III scores at 180 minutes were reduced (i.e., motor performance improved) on average by $32 \%$ after LD+DDCI, by $16 \%$ after MP-Ld, and by $50 \%$ after MP+DDCI. Intake of the same dose of levodopa from either MP (MP-Hd) or the pharmaceutical preparation (LD-DDCI) provided similar motor responses at both time points.

Mean latency to on with MP-Hd was significantly shorter than with LD+DDCI $(p=0.008)$, whereas it was similar to LD+DDCI with LD-DDCI, MP$\mathrm{Ld}$, and MP+DDCI.

Mean duration of the on state was 25\% longer with MP-Hd than with LD+DDCI (221 vs 177 minutes, $p<0.001$ ), while it was similar with MP-Ld and LD+DDCI. The comparison of MP and similar doses of pharmaceutical preparations of levodopa with and without DDCI (MP+DDCI vs $\mathrm{LD}+\mathrm{DDCI}$ and MP-Hd vs LD-DDCI, respectively) showed $20 \%$ shorter on duration with MP ( $p<0.05$ for both).

Safety. Dyskinesias at 90 minutes were fewer with MP-Hd and LD-DDCI than with LD+DDCI. We did not find any differences among LD+DDCI, MP-Ld, and MP+DDCI (table e-2).

No major AEs occurred and no patients dropped out of the study protocol. When compared to $\mathrm{LD}+\mathrm{DDCI}$, the number of AEs was significantly lower with MP-Ld, and we found a trend towards reduced AEs with MP-Hd (table 2). AEs most commonly occurred within 30-45 minutes after treatment administration and lasted $<15$ minutes, except for 4 patients, who reported AEs lasting $>90$ minutes after LD-DDCI. Despite similar levodopa dose, LD-DDCI was associated with more frequent AEs than MP-Hd, and it was the only treatment associated with prolonged AEs. There was no difference among the active treatments in terms of change in blood pressure and heart rate between off and on state (table e-3). Placebo intake was different from all the other arms, as it did not induce any change from baseline.

Pharmacokinetics. We additionally explored the relationship between motor response to treatments and levodopa pharmacokinetics in 4 Italian patients with PD (this analysis was not feasible in Bolivia due to the lack of adequate laboratory facilities and technical experience). Details are provided as supplementary material.

Levodopa plasma concentration and clinical response to $\mathrm{MP}, \mathrm{LD}+\mathrm{DDCI}$, and $\mathrm{LD}-\mathrm{DDCI}$ are presented as e-Results, table e- 4 , and figure e-2.

DISCUSSION This study demonstrates that the acute intake of MP powder at both high and low dose is noninferior to dispersible levodopa/benserazide in terms of all efficacy and safety outcome measures.

Both low-dose and high-dose MP were superior to placebo. Low-dose MP was associated with a motor response that was equivalent to the response to 


\section{Table 1 Demographic and general clinical features of patients with Parkinson disease (PD) at baseline}

\begin{tabular}{|c|c|}
\hline & $\begin{array}{l}\text { Patients with } \\
\text { PD }(n=18)\end{array}$ \\
\hline \multicolumn{2}{|l|}{ Features } \\
\hline Male sex, $n(\%)$ & 13 (72.2) \\
\hline Age, y & $61.8(9.1)$ \\
\hline Age at onset, y & $52.1(9.5)$ \\
\hline Body weight, kg & $75.1(16.7)$ \\
\hline Disease duration, y & 9.8 (3.0) \\
\hline $\begin{array}{l}\text { Disease duration at initiation of } \\
\text { levodopa therapy, } y\end{array}$ & $1.9(1.4)$ \\
\hline $\begin{array}{l}\text { Disease duration at initiation of } \\
\text { chronic MP therapy, } y^{a}\end{array}$ & $7.8(4.0)$ \\
\hline $\begin{array}{l}\text { Patients presenting tremor-dominant } \\
\text { phenotype }\end{array}$ & $10(55.6)$ \\
\hline UPDRS part I & $2.3(2.0)$ \\
\hline UPDRS part II: off & $16.1(6.5)$ \\
\hline UPDRS part III: off & $37.8(11.1)$ \\
\hline $\begin{array}{l}\text { UPDRS part IV: dyskinesias (items } \\
\text { 32-34) }\end{array}$ & $1.4(1.2)$ \\
\hline UPDRS part IV: off (items 36-39) & $4.2(2.2)$ \\
\hline Hoehn \& Yahr stage: off & $2.6(0.6)$ \\
\hline \multicolumn{2}{|l|}{ Motor complications } \\
\hline Duration of motor fluctuations, $y$ & 5.4 (3.1) \\
\hline $\begin{array}{l}\text { Duration of on time on current home } \\
\text { therapy, } \min ^{b}\end{array}$ & $158(58)$ \\
\hline $\begin{array}{l}\text { Disease duration at onset of motor } \\
\text { fluctuations, } y\end{array}$ & $5.4(2.0)$ \\
\hline $\begin{array}{l}\text { Levodopa duration at onset of motor } \\
\text { fluctuations, y }\end{array}$ & $3.6(2.1)$ \\
\hline $\begin{array}{l}\text { Disease duration at onset of } \\
\text { dyskinesias, } y\end{array}$ & $6.7(1.9)$ \\
\hline $\begin{array}{l}\text { Levodopa duration at onset of } \\
\text { dyskinesias, y }\end{array}$ & $5.1(2.3)$ \\
\hline \multicolumn{2}{|l|}{ Therapy } \\
\hline Total LEDD, $\mathrm{mg} / \mathrm{d}^{\mathrm{c}}$ & 1,457 (858) \\
\hline Duration of levodopa therapy, y & 7.9 (3.6) \\
\hline Levodopa dose, mg/d & 897 (331) \\
\hline Levodopa dose including MP, $\mathrm{mg} / \mathrm{d}^{\mathrm{d}}$ & $1,343(917)$ \\
\hline $\begin{array}{l}\text { Levodopa dose weight-adjusted, } \\
\mathrm{mg} \cdot \mathrm{kg}^{-1} \cdot \mathrm{day}^{-1 \mathrm{~d}}\end{array}$ & $19.3(11.2)$ \\
\hline Patients on stable MP therapy, $\mathrm{n}(\%)$ & $8(44.4)$ \\
\hline Patients on dopamine agonists, $\mathrm{n}(\%)$ & $11(61.1)$ \\
\hline Dopamine agonist dose, LEDD/d, mg & $279(57)$ \\
\hline $\begin{array}{l}\text { Duration of dopamine agonist } \\
\text { therapy, } y\end{array}$ & $5.6(2.0)$ \\
\hline Patients on iMAO-B, n (\%) & $0(0)$ \\
\hline Patients on iCOMT, n (\%) & $1(5.6)$ \\
\hline Patients on amantadine, $\mathrm{n}(\%)$ & $8(44.4)$ \\
\hline Patients on anticholinergics, $\mathrm{n}(\%)$ & $1(5.6)$ \\
\hline
\end{tabular}

Continued

\section{Table 1 Continued}

\section{Patients with} PD ( $n=18)$

Stable MP therapy ${ }^{\text {a }}$

Duration of MP therapy, y, median (IQR) 3.5 (2.8-4)

Current MP daily dose, g/d $35.6(20.7)$

Maximum MP single dose achieved, mg 11.1 (3.8)

Maximum MP daily dose achieved, $\mathrm{mg} / \mathrm{d} \quad 33.2$ (8.5)

Abbreviations: iCOMT = catechol-O-methyltransferase inhibitors; iMAO-B = monoamine oxidase type B inhibitors; IQR = 25-75 interquartile range; LEDD = levodopa-equivalent daily dose ${ }^{32}$; MP = Mucuna pruriens; UPDRS = Unified Parkinson's Disease Rating Scale.

Data are reported as mean (SD), unless otherwise specified. ${ }^{a}$ Calculated on the subgroup of patients who were on stable MP therapy before the initiation of the present study ( $\mathrm{n}=8$ ).

${ }^{b}$ Excluding patients who were on MP therapy.

${ }^{c}$ Levodopa/DDCl + levodopa contained in MP (calculated as $5.7 \%$ of MP weight) + dopamine agonist daily dose in LEDD.

${ }^{d}$ Including levodopa/DDCl and levodopa contained in MP.

levodopa/benserazide, while high-dose MP induced a qualitatively better motor response than $\mathrm{LD}+\mathrm{DDCI}$ along with longer duration of the on state by about 45 minutes and fewer dyskinesias. The latency to on was shorter with high-dose MP than with LD+DDCI (as also reflected by the shorter average $t_{\max }$ values obtained in the independent sample). This is likely to reflect a shorter latency with MP rather than a delayed one with $\mathrm{LD}+\mathrm{DDCI}$, as our results related to the mean latency to on with levodopa/benserazide are consistent with previous reports. ${ }^{22}$ Taken as a whole, efficacy data with MP powder from roasted seeds are in line with previous preclinical/clinical studies with MP extract (using pharmacologic processing), all consistently reporting a shorter latency to on $^{7}$ and longer on duration with reduced dyskinesias as compared to $\mathrm{LD}+\mathrm{DDCI}^{7-9}$ On the one hand, the longer duration of motor response may depend upon the higher levodopa dose administered; on the other, the significantly shorter latency to on suggests that MP is unlikely to act as an extended-release levodopa preparation. Are these encouraging clinical effects of MP related to some intrinsic properties that are independent of levodopa ${ }^{9}$ or merely due to the intake of levodopa alone? The latter hypothesis is the most probable, as the clinical response to $\mathrm{LD}-\mathrm{DDCI}$ overlapped with the effects of high-dose MP in terms of prolonged on state with reduced dyskinesias as compared to $\mathrm{LD}+\mathrm{DDCI}$. The present findings on MP closely remind us of the clinical trials that followed the development of the first peripheral DDCI in $1967,{ }^{19}$ demonstrating that the use of levodopa either alone or combined with DDCIs resulted in an overall similar efficacy on 


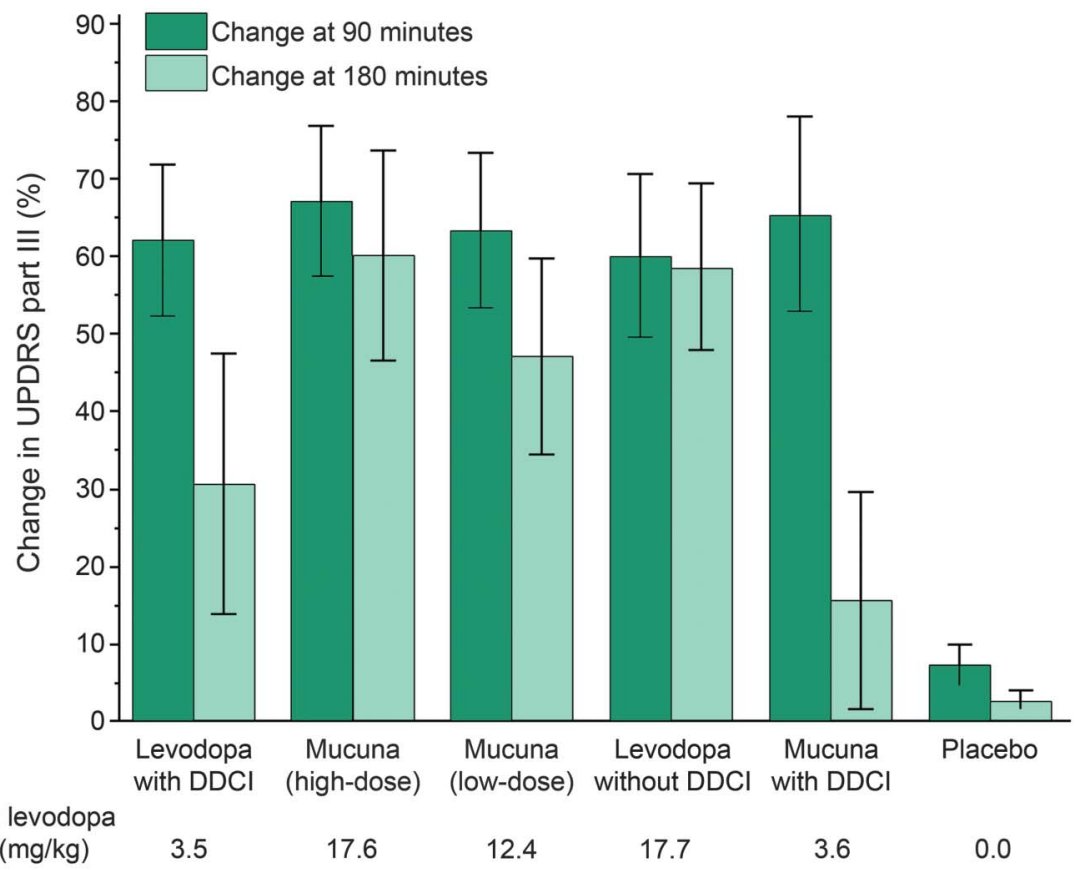

$\mathrm{DDCl}=$ dopa-decarboxylase inhibitor.

motor symptoms. ${ }^{13,16-19}$ Interestingly, the adjunct of DDCIs was reported to yield earlier and more severe dyskinesias despite the $60 \%-80 \%$ reduction in levodopa dose. ${ }^{14-19,23-26}$ These observations may contribute to explain the lower rates of dyskinesias we found with levodopa alone (MP-Hd and LD-DDCI) than with combined levodopa plus DDCI (LD+DDCI and $\mathrm{MP}+\mathrm{DDCI})$, despite the higher levodopa dose. In this scenario, the history of levodopa therapy before the introduction of $\mathrm{DDCIs}^{19}$ may teach us how to use MP-based therapy to lead low-income countries out of their current prelevodopa era. ${ }^{2-4}$
The safety profile of acute intake of MP powder was more favorable than the safety profiles of LD+DDCI and LD-DDCI. First, both low-dose and high-dose MP was associated with fewer AEs than LD+DDCI. Our findings are in line with previous studies showing that, despite the reduction in daily levodopa dose, the addition of DDCIs had either no effect or even led to worsening of hypotension, psychiatric disturbances, and dyskinesias to a greater extent than levodopa alone. ${ }^{16,25,27-30} \mathrm{We}$ found no differences among the active arms in terms of the cardiovascular profile, with an approximate

\begin{tabular}{|c|c|c|c|c|c|c|c|c|}
\hline \multicolumn{9}{|c|}{ Table 2 Frequency and type of adverse events } \\
\hline \multirow[b]{2}{*}{ Treatment arm } & \multicolumn{6}{|c|}{ Adverse event, $n$} & \multicolumn{2}{|l|}{$p$ Value ${ }^{a}$} \\
\hline & Any $^{\mathrm{a}}$ & Nausea & Somnolence & Dizziness & Psychiatric & Prolonged ${ }^{a, b}$ & $\begin{array}{l}\text { Compared to } \\
\text { high-dose MP }\end{array}$ & $\begin{array}{l}\text { Compared to } \\
\text { low-dose MP }\end{array}$ \\
\hline MP high-dose & 2 & 2 & 1 & 0 & 0 & 0 & - & 1 \\
\hline MP low-dose & 1 & 1 & 1 & 0 & 0 & 0 & 1 & - \\
\hline $\mathrm{MP}+\mathrm{DDCl}$ & 1 & 0 & 1 & 0 & 0 & 0 & 1 & 1 \\
\hline Levodopa + DDCI & 7 & 0 & 6 & 1 & 0 & 0 & 0.063 & 0.031 \\
\hline Levodopa without DDCI & 12 & 6 & 9 & 3 & 1 & 4 & 0.006 & 0.001 \\
\hline Placebo & 1 & 0 & 1 & 0 & 0 & 0 & 1 & 1 \\
\hline Total number of events & 24 & 9 & 19 & 4 & 1 & 4 & - & - \\
\hline
\end{tabular}

Abbreviations: $\mathrm{DDCl}$ = dopa-decarboxylase inhibitor (Benserazide); MP = Mucuna pruriens.

${ }^{a}$ Number of patients with one or more AEs.

${ }^{\mathrm{b}} \mathrm{AEs}$ lasting $>90$ minutes. 
20-25 $\mathrm{mm} \mathrm{Hg}$ drop and $10-15 \mathrm{~mm} \mathrm{Hg}$ drop in systolic and diastolic values, respectively (highest drops with LD+DDCI, albeit not significant). Previous trials on levodopa alone showed that a drop by $15-20 \mathrm{~mm} \mathrm{Hg}$ was common and that it was usually asymptomatic and transient. ${ }^{16,28}$ Second, high-dose MP induced significantly less AEs than a similar dose of pharmaceutical preparation of levodopa alone. Furthermore, prolonged AEs lasting longer than 90 minutes were recorded only after LD-DDCI intake, while the few AEs induced by MP were mild and transient. These data support the hypothesis of an intrinsically more favorable safety profile of MP powder than pharmaceutical preparations of levodopa, regardless of the presence/absence of DDCIs.

The present cohort does not entirely represent the target population in low-income areas, because these patients had access to standard levodopa/carbidopa therapy. Although the DDCI plasma half-life is 3 hours, we cannot exclude a residual DDCI effect after the last intake of LD+DDCI, which usually occurred 12 to 14 hours before the challenge (corresponding to $4-4.5$ half-lives). ${ }^{7,31}$ If such effect was present, the intake of high-dose levodopa alone (MP-Hd and LD-DDCI) would have greatly increased the severity of dyskinesias. However, we found the opposite response, making a residual DDCI effect unlikely to have biased the present data. Furthermore, we included only patients with motor fluctuations and dyskinesias in order to provide a clear characterization of efficacy and safety endpoints. Multicenter studies on de novo patients with PD followed for long peri$\mathrm{ods}^{29}$ are needed to obtain practice guidelines for MP monotherapy.

What is the main issue that we expect with the intake of MP powder in the long term? The answer is tolerability. The safety of chronic intake of levodopa alone at high daily dosage (up to $16 \mathrm{~g} / \mathrm{d}$ ) was investigated for over a decade in more than 2,000 humans. ${ }^{1,16,26}$ The most common dose-limiting AEs of levodopa alone were gastrointestinal disturbances, which were usually transient ${ }^{15,17}$ and whose frequency and intensity were minimized by very slow titration schemes. ${ }^{126,29}$ Considering that an a priori estimate of levodopa content in individual MP samples will rarely be feasible in low-income areas, any MP-based therapy will inevitably require very slow titration schemes. These schemes are to be defined taking the range of levodopa concentrations into consideration (from $4 \%$ to $6 \%$ in the majority of samples $\left.{ }^{6,11}\right)$, along with PD duration and body weight. Considering the major problems of availability/affordability of levodopa in low-income countries, ${ }^{2-4}$ it is conceivable that the benefits of MP-based therapy will overcome such issues in the long term, at least in those who would otherwise remain untreated. The patients who remain undertreated because of discontinuous access to marketed levodopa preparations may benefit from a combination of low-dose LD+DDCI and MP to spare such supplies (as is currently the case in Santa Cruz, Bolivia).

MP was noninferior to levodopa/benserazide in terms of all efficacy and safety outcome measures. The clinical response to MP was similar to the effects of a pharmaceutical preparation of levodopa alone at similar doses, with less AEs. MP could be a sustainable alternative to marketed levodopa for indigent individuals with PD in low-income countries, provided that it is tolerated in the long term.

\section{AUTHOR CONTRIBUTIONS}

Study concept and design: Drs. Cilia and Pezzoli. Acquisition of data: Drs. Cilia, Laguna, Cassani, and Pozzi. Analysis and interpretation of data: Drs. Cilia, Laguna, Cassani, Cereda, Contin, Barichella, and Pezzoli. Drafting of the manuscript: Drs. Cilia. Critical revision of the manuscript: Drs. Cilia, Laguna, Cassani, Cereda, Isaias, Contin, Barichella, and Pezzoli. Statistical analysis: Drs. Cereda. Obtained funding: Dr. Pezzoli. Administrative, technical, or material support: Drs. Laguna and Pezzoli. Study supervision: Dr. Pezzoli.

\section{ACKNOWLEDGMENT}

The authors thank all the patients involved in the study and their families; Dr. Alice Leporini for help in the pharmacokinetics section of the study; and the medical writer Jennifer S. Hartwig for assistance in editing the manuscript.

\section{STUDY FUNDING}

This study was funded by the "Fondazione Grigioni per il Morbo di Parkinson," Milan, Italy.

\section{DISCLOSURE}

The authors report no disclosures relevant to the manuscript. Go to Neurology.org for full disclosures.

Received November 7, 2016. Accepted in final form May 5, 2017.

\section{REFERENCES}

1. Cotzias GC, Van Woert MH, Schiffer LM. Aromatic amino acids and modification of parkinsonism. N Engl J Med 1967;276:374-379.

2. Cilia R, Akpalu A, Sarfo FS, et al. The modern prelevodopa era of Parkinson's disease: insights into motor complications from sub-Saharan Africa. Brain 2014;137: 2731-2742.

3. Dotchin C, Msuya O, Kissima J, et al. The prevalence of Parkinson's disease in rural Tanzania. Mov Disord 2008; 23:1567-1672.

4. Mokaya J, Dotchin C, Gray WK, Hooker J, Wlaker RW. The accessibility of Parkinson's disease medication in Kenya: results of a national survey. Mov Disord Clin Pract 2016;3:376-381.

5. Diamond SG, Markham CH, Hoehn MM, McDowell FH, Muenter MD. Multi-center study of Parkinson mortality with early versus later dopa-treatment. Ann Neurol 1987;22:8-12.

6. Cassani E, Cilia R, Laguna J, et al. Mucuna pruriens for Parkinson's disease: low-cost preparation method, laboratory measures and pharmacokinetics profile. J Neurol Sci 2016;365:175-180. 
7. Katzenschlager R, Evans A, Manson A, et al. Mucuna pruriens in Parkinson's disease: a double blind clinical and pharmacological study. J Neurol Neurosurg Psychiatry 2004;75:1672-1677.

8. Lieu CA, Kunselman AR, Manyam BV, Venkiteswaran K, Subramanian T. A water extract of Mucuna pruriens provides long-term amelioration of parkinsonism with reduced risk for dyskinesias. Parkinsonism Relat Disord 2010;16:458-465.

9. Lieu CA, Venkiteswaran K, Gilmour TP, et al. The antiparkinsonian and antidyskinetic mechanisms of Mucuna pruriens in the MPTP-treated nonhuman primate. Evid Based Complement Alternat Med 2012;2012:840247.

10. Ovallath S, Deepa P. The history of parkinsonism: descriptions in ancient Indian medical literature. Mov Disord 2013;28:566-568.

11. Kasture S, Mahalaxmi Mohan M, Kasture V. Mucuna pruriens seeds in treatment of Parkinson's disease: pharmacological review. Orient Pharm Exp Med 2013;13: 165-174.

12. Hughes AJ, Daniel SE, Kilford L, Lees AJ. Accuracy of clinical diagnosis of idiopathic Parkinson's disease: a clinico-pathological study of 100 cases. J Neurol Neurosurg Psychiatry 1992;55:181-184.

13. Calne DB, Reid JL, Vakil SD, et al. Idiopathic parkinsonism treated with an extracerebral decarboxylase inhibitor in combination with levodopa. Br Med J 1971;3:729-732.

14. Mars H. Modification of levodopa effect by systemic decarboxylase inhibition. Arch Neurol 1973;28:91-95.

15. Jaffe ME. Clinical studies of carbidopa and L-DOPA in the treatment of Parkinson's disease. In: Yahr MD, ed. Advances in Neurology, vol 2. New York: Raven; 1973: 161-172.

16. Barbeau A. Treatment of Parkinson's disease with L-DOPA and Ro 4-4602: review and present status. In: Yahr MD, ed. Advances in Neurology, vol 2. New York: Raven; 1973.

17. Marsden CD, Parkes JD, Rees JE. A year's comparison of treatment of patients with Parkinson's disease with levodopa combined with carbidopa versus treatment with levodopa alone. Lancet 1973;2:1459-1462.

18. Fahn S. "On-off" phenomenon with levodopa therapy in Parkinsonism: clinical and pharmacologic correlations and the effect of intramuscular pyridoxine. Neurology 1974; 24:431-441.

19. Gershanik OS. Improving L-dopa therapy: the development of enzyme inhibitors. Mov Disord 2015;30:103-113.

20. Fahn S, Elton RL; UPDRS Program Members. Unified Parkinson's Disease Rating Scale. In: Fahn S, Marsden
CD, Goldstein M, Calne DB, eds. Recent Developments Parkinson's Disease, vol 2. Florham Park: Macmillan Healthcare Information; 1987:153-163, 293-304.

21. Guy W. Abnormal involuntary movement scale: ECDEU Assessment Manual for Psychopharmacology. Washington, DC: US Government Printing Office; 1976:534-537.

22. Merello M, Pikielny R, Cammarota A, Leiguarda R. Comparison of subcutaneous apomorphine versus dispersible madopar latency and effect duration in Parkinson's disease patients: a double-blind single-dose study. Clin Neuropharmacol 1997;20:165-167.

23. Papavasiliou PS, Cotzias GC, Düby SE, Steck AJ, Fehling C, Bell MA. Levodopa in parkinsonism: potentiation of central effects with a peripheral inhibitor. N Engl J Med 1972;286:8-14.

24. Chase TN, Watanabe AM. Methyldopa hydrazine as an adjunct to L-dopa therapy in parkinsonism. Neurology 1972;22:384-392.

25. Schwartz AM, Olanow CW, Spencer A. A double-blind controlled study of MK-486 in Parkinson's disease. Trans Am Neurol Assoc 1973;98:301-303.

26. Cotzias GC, Papavasiliou PS, Gellene R. Modification of parkinsonism: chronic treatment with L-dopa. N Engl J Med 1969;280:337-345.

27. Watanabe AM, Chase TN, Cardon PV. Effect of L-dopa alone and in combination with an extracerebral decarboxylase inhibitor on blood pressure and some cardiovascular reflexes. Clin Pharmacol Ther 1970;11:740-746.

28. Calne DB, Petrie A, Rao S, Reid JL, Vakil SD. Action of L-methyldopa-hydrazine on the blood pressure of patients receiving levodopa. Br J Pharmacol 1972;44:162-164.

29. Marsden CD, Barry PE, Parkes JD, Zilkha KJ. Treatment of Parkinson's disease with levodopa combined with L-alpha-methyldopa hydrazine, an inhibitor of extracerebral DOPA decarboxylase. J Neurol Neurosurg Psychiatry 1973;36:10-14.

30. Leibowitz M, Lieberman A. Comparison of dopa decarboxylase inhibitor (carbidopa) combined with levodopa and levodopa alone on the cardiovascular system of patients with Parkinson's disease. Neurology 1975;25: 917-921.

31. Khor SP, Hsu A. The pharmacokinetics and pharmacodynamics of levodopa in the treatment of Parkinson's disease. Curr Clin Pharmacol 2007;2:234-243.

32. Tomlinson CL, Stowe R, Patel S, Rick C, Gray R, Clarke CE. Systematic review of levodopa dose equivalency reporting in Parkinson's disease. Mov Disord 2010;25: 2649-2653. 


\section{Neurology}

\section{Mucuna pruriens in Parkinson disease: A double-blind, randomized, controlled, crossover study \\ Roberto Cilia, Janeth Laguna, Erica Cassani, et al.}

Neurology 2017;89;432-438 Published Online before print July 5, 2017

DOI 10.1212/WNL.0000000000004175

\section{This information is current as of July 5, 2017}

\section{Updated Information \& Services}

Supplementary Material

\section{References}

Subspecialty Collections

Permissions \& Licensing

Reprints including high resolution figures, can be found at:

http://n.neurology.org/content/89/5/432.full

Supplementary material can be found at:

http://n.neurology.org/content/suppl/2017/07/05/WNL.0000000000004 175.DC1

http://n.neurology.org/content/suppl/2017/07/05/WNL.0000000000004 175.DC2

This article cites 29 articles, 7 of which you can access for free at: http://n.neurology.org/content/89/5/432.full\#ref-list-1

This article, along with others on similar topics, appears in the following collection(s):

\section{Class II}

http://n.neurology.org/cgi/collection/class_ii

Clinical trials Randomized controlled ( $\overline{\mathbf{C} O N S O R T}$ agreement)

http://n.neurology.org/cgi/collection/clinical_trials_randomized_control led_consort_agreement

Parkinson's disease/Parkinsonism

http://n.neurology.org/cgi/collection/parkinsons_disease_parkinsonism

Information about reproducing this article in parts (figures,tables) or in its entirety can be found online at:

http://www.neurology.org/about/about_the_journal\#permissions

Information about ordering reprints can be found online:

http://n.neurology.org/subscribers/advertise

Neurology ${ }^{\circledR}$ is the official journal of the American Academy of Neurology. Published continuously since 1951, it is now a weekly with 48 issues per year. Copyright Copyright ( 2017 The Author(s). Published by Wolters Kluwer Health, Inc. on behalf of the American Academy of Neurology. All rights reserved. Print ISSN: 0028-3878. Online ISSN: 1526-632X.

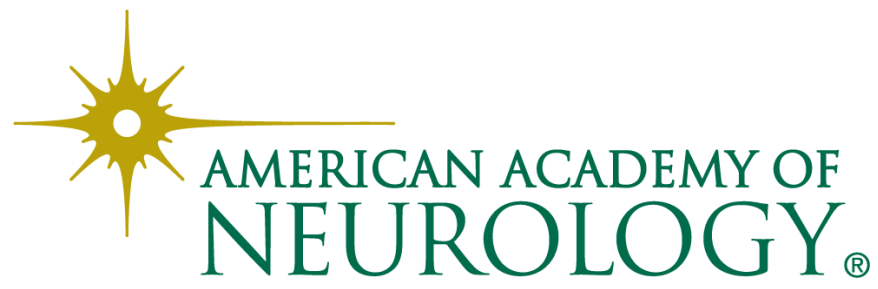

